\title{
Nonlinear Diffusion Filtering Method Based on Wavelet Image
}

\author{
Zhao Xiaofeng \\ Shandong Women's University \\ zhxf0929@sina.com
}

\begin{abstract}
In this paper, on the basis of the anisotropic diffusion mechanism, analyzes emphatically represented by $P$ - M model of diffusion filter principle of several kinds of nontinear diffusion model, and their respective characteristics and problems. In-depth analysis of the nonlinear diffusion model, the threshold and termination mechan su of combining image geometric structure feature and visual information (gradient, brightness, contrast, structural information), in view of the existing nonlinear diffusion filtering model, the diffusion coefficient depends on the gradient and the problem that the sulsceptible to noise interference, presents a fidelity term used in image denoising and restoration contain nonlinear wavelet diffusion model, the theoretical analysis and experimental results show that this method is compared with other diffusion model whlle denoising can keep image edges and details characteristics, image visual effect is betier.
\end{abstract}

Keywords: Quaternion analyfic signal, Qhatemion wavelettransform $(Q W T)$, Image denoising, Donoho's threshold

\section{Introduction}

Image is the main medium of information transmission and the foundation of the human visual image information processing technology is access to the necessary technical means, the visual message. As a branch of signal processing, image processing and understanding has become a modern one of the main technology of electronic information science and technology, the modern economic and social development and peoples work life to produce a significant and profound influence. Digital image processing, digital image processing), also known as the computer image processing, in the $1960 \mathrm{~s}$ with the development of computer technology and the establishment and development of Fourier transform theory of a new technology [1-2], information processing and computer vision is an important part of. Computer image processing, the use of computer on economic construction, social development and hunan life and scientific research in the digital processing of visual information. Stand in the perspective of application, the main task of the image processing includes three aspects: (1) the mathematical theory and other technical means, enhance the image visual effect. For example, through to the image brightness, color, contrast, and a series of mathematical transformation, inhibition of noise in the image, highlight the need to some of the characteristics of information, improve the image visual quality. (2) To extract the image contains some useful information, including frequency characteristics, grayscale characteristics, color characteristics, boundary and regional characteristics, texture and geometric features, etc., for the subsequent image understanding, analysis and application, the study of machine vision. (3) The use of information and

1. Shandong Province natural sciences fund project. ZR2012FL04. Design and Arithmetic Research of Image Sparse Dictionary.

2. Shandong Province natural sciences fund project. ZR2013FL024. Countour grouping method based on particle filter and its application in image retrieval ISSN: 1975-0080 IJMUE Copyright (C) 2014 SERSC 
communication technology, the research of image information encoding, compression, storage, and transmission theory and method, improve the efficiency of image storage and transmission, and security.

Image in the acquisition, storage, transmission and processing of each link, due to various noise interference, resulting in quality reduction. These noises can cause the image blur, more serious may cause the lack of image features, image analysis work after it is very difficult to provide useful information. Therefore, for many specific image processing tasks, for example, image segmentation, target recognition and edge detection, image denoising is the first step needs to be done, its purpose is to eliminate the influence of noise on image quality is a classic problem in image restoration. Image restoration is refers to the use of degeneration phenomenon of some kind of a priori knowledge to reconstruct or recover was degraded image. Image in the process of denoising, however, often fail to balance the image detail features keep and denoising degree, the relationship between the denoising of inevitable cause edge blur at the same time, the detailed features missing such as a problem. And the high frequency part of image, for example, details, edge, etc., is a sepsitive area of human vision, and the image edge information exists in most of the details. Therefore, there are many scholars studying denoising while keeping the edge and detail characteristics of the model and algorithm, and emerged many effective new model.

Partial differential equation is applied in image restoration mainly includes two forms: based on partial differential equation of conduction coefficient of anisotropic diffusion model and based on the variational principle of energy functional optimization model. The former according to specific image processing tasks and control the conduction coefficient, analysis and designdirection of diffusion and spread, so as to realize the different diffusion of regionalinternal and regional boundaries, to rein in at the same time keep the image edge details characteristics of the noise. The latter through the image of grey value of pixels to establish energy functionals, the optimization process of the euler Lagrange equation as a partial differential equation of diffusion process. By adjusting the format of the energy functional, achieve the goal of image denoising and edge. Although the starting point of thinking of the two methods are different, but deduces partial differential model has many similarities. This article mainly discusses the image diffusion principle based on partial differential equation, the typical model analysis, this paper offers a new model parameter estimation and optimization method of P - M; Put forward a kind of based on wavelet transform and the image of the fidelity term containing nonlinear diffusion filtering model, and through experiment verifies the superiority of the model [3-6].

Images are usually made of smooth area, edge, texture, corner regions of different chacacteristics, such as. How to depict and judge these areas, it is a pressing problem in image processing. Image processing and analysis method based on partial differential equations can be according to the local neighborhood information to determine a pixel in the image features of that point, and then automatically choose the reasonable processing mechanism. Compared with other modern image processing method, partial differential equation method is the biggest difference is that the anisotropic diffusion performance. Therefore, it can in denoising (smooth) or reconstruction of homogenous area at the same time, maintain or enhance the details such as edge, texture features [7].

\section{Related Works}

Noise is unavoidable and unpredictable random error, the noise of the randomness of only by probability statistics method to estimate, usually with its numerical 
characteristics, such as described by the mean value, variance, etc. According to the causes, image noise can be divided into external noise caused by external disturbance and internal noise caused by electrical equipment system internal. According to the statistical characteristics of image noise and can be divided into smooth change over time and does not change with time of non-stationary noise. Koenderink and Witkin in the field of image processing, introduces the concept of scale space, set up the image multi-scale space representation and analysis theory, known as the partial differential equations in image processing field made substantial progress. Hummel, 1986 pointed out that thermal diffusion equation is not only can be used to generate the multi-scale space of parabolic equation, and prove any satisfy the maximum principle of evolution equation can define a multi-scale space. Due to the partial differential equation from the continuous domain, which can be associated with some physical process and therefore can be explained from the point of view of movement, image processing and analysis. Thermal diffusion equation is the earliest diffusion equation is used for solving the problem of image, is also a classical partial differential equation for inage processing. In physics, the uneven distribution of impurity concentration of medium, the impurities from the high concentration area to the low concentration area migration process called diffusion. Similarly, if the uneven distribution of medium temperature, high temperature to low temperature area energy transfer process, is called the thermal diffusion. Thermal diffusion equation of image filtering, with the increase of the number of iterations and the increase of the scale parameter, the image is blurred, iteration terminates the pixel gray value will reach an average constant. To improve the disadvantages of this linear diffuston, according to human visual characteristics, structure features of the image, and is used to control the edge diffusion behavior, realize at the same time keep the edge details characteristics of denoising. Therefore, through constructing the gradhent as the independent variable of edge detection function as diffusion coefficient the smaller gradient diffusion coefficient (flat) area is large, is beneficial to remove noise, and in the large gradient region (edge) diffusion coefficient is small, to maintain the edge, this equation is known as linear inhomogeneous diffusion equation. But its shortcomings exist the following two aspects: first, according to the original image edge gradiem judgment, wrong appear very easily affected by noise; Second, the diffusion coefficient in edge area is very small, even tend to be 0 , equivalent to basic not to deal with the noise in edge area, edge area denoising effect is not ideal. Therefore, the researchers on the basis of many improved model is put forward, in order to achieve at the same time keep the edge details such as features of denoising [8-10].

\subsection{Anisotropic Diffusion}

Images often contain different characteristics in the area, for example, smooth homogeneous area, regional boundary, angular point, texture, etc. Model of image processing based on partial differential equations can be according to the local neighborhood pixels in the image information to determine the pixel region characteristic information, and then automatically choose reasonable diffusion behavior, is implemented in different regions of the anisotropic diffusion, which is a partial differential equation with other image processing methods of the biggest differences. , therefore, be able to smooth (noise) at the same time, maintain or enhance edge feature area. To do this, you can consider using nonlinear partial differential equation model of operator to denoising. Its main idea is in the process of diffusion to set a "diffusion coefficient", make it automatically adjust according to the local characteristics of image, 
that is to say, when in flat areas of the image, can automatically increase diffusion coefficient and makes the denoising performance improvements, and near the image edge, diffusion coefficient decreases, and automatically protect the edges. Just rely on the gradient information of the original image, however, to judge the characteristics of the pixel area, often due to the interference of noise, and the image edge, corner, texture and other important features to produce, in order to solve this problem, Perona and Malik anisotropic diffusion equation in 1990 proposed (hereinafter referred to as $\mathrm{P}-\mathrm{M}$ equation). $\mathrm{P}$ - M diffusion equation using directional diffusion instead of keep on the edge of the isotropic diffusion in the thermal diffusion, and can according to regional structural features in the image automatically adjust the diffusion coefficient, carried out in heterogeneous diffusion image different feature regions. But $\mathrm{P}-\mathrm{M}$ equation/itself is sick, can't guarantee the uniqueness of solution. For this purpose, the Frepch scholar Catte scholars with gaussian kernel and the image as a convolution operation, to smooth the image, and then take the gradient model to estimate the image edge information, the establishment of a well-posed diffusion equation. Torkamani Azar and Tait scholars used to optimize symmetry index such as filter to smooth the image, and then to the gradient model to estimate the image edge information. Catte method and Torkamani Azar method the basic idea is by suppressing noise interference, accurate as far as possible to extract the image edge character intormation. in order to better control the diffusion behavior of $\mathrm{P}-\mathrm{M}$ equation. Both estimation method, however, there is a smooth scale parameter selection difficult problem. In fact the two methods are isotropic diffusion of image that confrary to the jdeas of the edge is smooth.

Alvarez scholars from geometric point of view, this paper proposes a diffusion model based on mean curvature (mean durvaturentotion, MCM), solves the well-posedness of P - M model. MCM model has good geometric meaning, and be able to stop the spread of the edge, edge structures. Later, they further proves that meet certain conditions of image scale space can be derived from the corresponding parabolic PDE, and put forward with affine invariance of multi-scale analysis equation. Weickert will structure tensor (structure tensor, ST) Combined with $\mathrm{P}-\mathrm{M}$ equation, using tensor diffusion coefficient of eigenvalue structure in different directions, to control the spread of strength, is proposed based on structure tensor image coherence enhancing diffusion model, the model in the image at each pixel defines a coherent direction, along the direction of nonlinear diffusion to restore fracture linear structure, at the same time, also make the edge texture effectively suppress noise. In the use of traditional second order partial differential equation for image denoising, it's easy to have a staircase effect, in order to overcome this problem, in 2000, You scholars such as high order partial differential equation model is put forward. In this model, the image gradient $\nabla \mathrm{u}$ $\nabla 20$ form instead. The model at the same time, on the edge of denoising and effectively avoid the phenomenon of the block effect, but its defect is obvious: in the filter out the noise of the smooth area and eliminate the staircase effect at the same time, will lead to a large number of images generated after filtering spots, make the image is uneven phenomenon.

\subsection{P - M Equation of Parameter Estimation and Optimization}

How to obtain high quality in the spread of the stability of the image, is the most concern in the diffusion process, and the final image quality and selection of various parameters in nonlinear diffusion equation has a direct relationship. For P - M diffusion equation, for example, how to select the most optimal parameters is not yet formed an 
effective method, main is estimated by experience. In order to facilitate analysis, the $\mathrm{P}$ $M$ model of two types of diffusion coefficient to write the following:

$$
\begin{gathered}
g(|\nabla u|)=\exp \left(-(|\nabla u| / k)^{2}\right) \\
g(|\nabla u|)=\frac{1}{1+(|\nabla u| / k)^{2}}
\end{gathered}
$$

Although the two types of diffusion coefficient have similar filtering effect, but their diffusion mechanism and the effect is different. Type (1) priority diffusion high contrast boundary, type (2) the diffusion of border area. No matter choose which kinds of diffusion function $|\nabla u|>k$, at that time, the diffusion of power is weak, border details characteristics are kept; On the contrary, when $|\nabla u|<k$ the spread of power is stronger, smoothing effect is obvious. That is to say, in the process of diffusion the selection of the $\mathrm{k}$ value is very important, it determines which areas are treated as edges, what areas are treated as plain area. Corresponds to the edge of the area, diffusion is abate, on the contrary, $|\nabla u|>k$ diffusion. Therefore, in the $\mathrm{P}-\mathrm{M}$ model and its improved model, how to select the gradient threshold $\mathrm{k}$ to the filtering effeet of algorithm play a crucial role.

Perona and Malik advice with Canny noise estimation method to determine the gradient threshold $\mathrm{k}$. The calculation of the ctrmulative histogram after each iteration, the general of $\mathrm{k}$ value for the total pixets $\sim \mathrm{u} \mid$ value of $0.85 \sim 0.90$. In addition, for some particular image, can consider to manually adust the different threshold $\mathrm{k}$, since the childhood gradually adjusted to the satisfying effect. P - M model about the selection of parameter k, also attracted the attention of many researchers. Among them, the Voci put forward by the two ways of estimated parameter k: morphology method and the method based on $\mathrm{P}$ - norm [6].K estimation method based on morphology of large amount of calculation, and with $\mathrm{p}$-norm estimates can guarantee $\mathrm{k}$ decreased, and the small amount of calculation. If image process, with the increase of the number of iterations, edge gradient modulus value decreased gradually, in order to guarantee smooth power not too strong, so as to avoid the fuzzy edges and detail, then the gradient threshold $\mathrm{k}$ should also be reduced accordingly. In addition, because the conditions for preserving $|\nabla \mathrm{u}|>\mathrm{k}$, it also requires the gradient modulus reduce at the same time the corresponding threshold value k. Voci puts forward two methods rely on the gradient to metric space details, local details description is incomplete, so in a smooth and details remain poor. Literature [7] proposed the diffusion time scale function is estimated using the regular parameter method. [8] Advice for diffusion time scale reduction function to reduce the sensitivity of the model of diffusion time scale. [9] The noise and are assumed to be uncorrelated, noise and the signal correlation coefficient reaches the minimum time as a stop time scales. But no matter what kind of method need to know in advance the noise variance to effectively estimate, and often know little about the noise characteristics in practical application. In addition, these methods are not considering structural features of the image itself with poor adaptability for different images, so that the effectiveness of this kind of method is greatly reduced. In nonlinear diffusion model, involves two important parameters: the threshold and spread the stop time. These two parameters selection of appropriate or not directly related to the smooth image quality, so it is necessary for the selected methods were studied.

How to choose the stop time is anisotropic diffusion equation and its various improvement equation are another important factor to consider, it has great influence on the effects of diffusion filter. If the diffusion time is too long, restored image will 
appear smooth, edge blur, detailed features missing, etc. On the other hand, if the diffusion time is too short, get rid of the noise can't very good, filtering effect is not good. Ideally, when the noise is removed, the diffusion is terminated, and the edge details such as characteristic information is completely preserved. In real application, it is hard to reach such an ideal state, therefore, in the process of image restoration is often compromise approach. In general, no noise pollution diffusion termination time USES the original image and the restored image of minimum mean square error (mse) to restrict and determined. But, in fact, we don't know the original noiseless image, so we must adopt other means to calculate diffusion stop time. Stein's SURE (Stein 's unbiased risk estimate) estimation method are often used to estimate the diffusion termination time, good results have been achieved. In addition, in the SURE Ramani and others method, inspired, proposed a don't need to know the MSE estimation method of the original image. In addition, studies have shown that the human eye when looking at an image, first to extract the image structure information, because information correlation between image pixels, the human eye judge of a picture drop quality, and the image quality, is more of a look at the structure of the image information and the structure of the original image is similar. Based on the above assumptions, [10] proposed the "structural similarity (structure similarity, SSIM)" the objective evaluation method based on the person's subjective feeling.

\section{Image Nonlinear Diffusion Filter based orWavelet Transform}

\subsection{Fidelity Term with Nonlinear Wavelet Diffúsion Model}

According to the previous analysis to know, on the one hand, P - M equation in the process of diffusion cannot accurately/distinguish between geometric structure characteristics of the image itself and noise caused by the gradient amplitude changes; On the other hand, the anisotropic partial differential diffusion equation itself is sick, can't guarantee the uniqueness of solution. Catte people such as classic filtering method was applied t6 P M model is improved, the well-posed nonlinear diffusion model is presented. Although Catte model experimental results is good, but the gaussian smoothing is equivalent to the image itself isotropic low-pass filtering, edge easy to cause extreme value point drift, which is contrary to the ideas of the anisotropic diffusion. In addition, the gaussian function of factor selection of sigma is very sensitive, greatly influenced the result of the image smooth. Ideal denoising and recovery strategy is at the same time, the denoising can be a very good image of the geometry lnformation, such as area, edge, Angle and internal texture details. The requirements to meet the following conditions: (1) be implemented within the flat area strong diffusion, has good smoothing effect; (2) in the image edge, only along the edge of tangential spread, to keep the edge; (3) in the image edges and sharp corners, introducing the fidelity term, to maintain the evolution of image and the original image similarity, prevent smooth along the edge of the tangential caused the loss of image geometry characteristic information. Based on the above thought, in view of the existing nonlinear diffusion filtering model, the diffusion coefficient depends on the gradient and the problem that the susceptible to noise interference, combined with the thought of literature [9], this section presents a fidelity term used in image denoising and restoration contain nonlinear wavelet diffusion model (nonlinear wavelet coursing together method, NWDM). 


$$
\frac{\partial u}{\partial t}=\operatorname{div}[g(W u) \nabla u]+\alpha\left(W u_{0}-W u\right)
$$

$\mathrm{Wu}, W u_{0}$ said image $\mathrm{u}$ and $u_{0}$ wavelet transform modulus square, for the fidelity term used to control the diffusion process of evolution de gree of approximation of image and the original image is a 2 sigma and noise intensity is inversely proportional to the control coefficient. $\mathrm{g}(\cdot)$ is still the smooth monotone decreasing function. Compared with $\mathrm{P}$ - M model, wavelet transform modulus square to replace the gradient mode in the $\mathrm{P}$ - M model. In other words, the spread of each point depends on the amount of that point in the wavelet transform modulus. Mainly use wavelet transform to extract the image edge information, so only to the image wavelet transform of the horizontal and vertical direction $W_{a}^{k} u(x, y)=\left\langle u(t, v), \varphi_{a, x, y}^{k}(t, v)\right\rangle \cdot k=1,2$ Of wavelet transform for the two direction, among them, and are respectively on the direction of horizontat and vertical two wavelet. The wavelet transform modulus square

$$
W_{a} u(x, y)=\left|W_{a}^{1} u(x, y)\right|^{2}+\left|\hat{W_{a}} u(x, y)\right|^{2}
$$

Reflects the image of $\mathrm{u}(\mathrm{x}, \mathrm{y})$ point of gray level transformation. In the image of the graded or flat area, Mau (x, y) has a smaller value; On the edge or singularity has great value, and the stronger the singularity, Mau $(x, y)$. In addition, the noise of the signal are mainly concentrated on the small scale wavelet transform coefficient, and with the increase of decomposition scale and apid attenuation And the signal wavelet transform coefficient increases with the increase of decomposition scale or unchanged. Therefore, from the image on the wavelet transform coeffieient of division can accurately reflect the image of the singularity, mutation (suich as the edge, etc.).

To verify NWDM model dénoising effect, in the given contains fidelity term P - M lambda model:

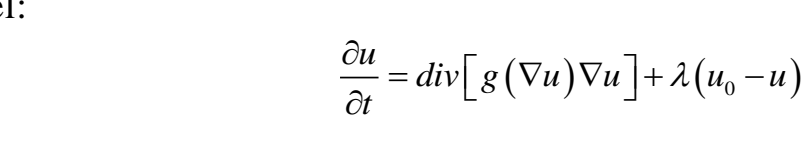

HMT model using hidden markov tree structure reflect the correlation of wavelet coefficients between the seale, the use of markov chain on the multi-scale modeling of wavelet coefficients corresponding to the number of hidden states. HMT the hidden states of wavelet coefficients of large and small, big state of wavelet coefficients with large variance fof gaussian distribution to model; Small state with small variance of the wavelet coefficients of gaussian model. Actual signal after quaternions wavelet transform coefficient between displays continuity and clustering characteristics, at the same time, the statistical results show that after the transformation of the coefficient of peak, long trailing edge density non-gaussian distribution characteristics. [7] Mentioned in the state of the two zero mean gaussian mixture model can be a good approximation of the actual image wavelet coefficient distribution. In this paper the Q - HMT model, each wavelet coefficients using the state of the two gaussian mixture model to represent, and each domain of wavelet coefficients corresponding to state variables it four variables on the next layer is linked together, the relationship between the coefficient of only by the father and son the state transition probability between state variables of the embodiment. Under normal circumstances, the state of two Gaussian mixture model of random probability density function of wavelet coefficient $\mathrm{W}$ consists of two parts: the probability of state variable $\mathrm{S}$ function $P_{S}(m)$ and the conditions of the Gaussian probability density function 
Where

$$
\begin{gathered}
f_{W \mid S}(W \mid S=m), \quad f_{W}(w)=\sum_{m=1}^{2} p_{s}(m) f_{W \mid S}(W \mid S=m) \\
f_{W \mid S}(W \mid S=m)=\frac{1}{\sqrt{2 \pi \sigma_{m}^{2}}} \exp \left(\frac{-\left(w-\mu_{m}\right)^{2}}{2 \sigma_{m}^{2}}\right)
\end{gathered}
$$

Given a set of scale for the wavelet coefficient of $J$, assumptions and respectively the wavelet coefficient of random variables and state variables, in which $\mathrm{I}=1, \ldots \mathrm{J}, \mathrm{J}=$ $1, \ldots \mathrm{N}$ ( $\mathrm{N}$ scale for the wavelet coefficient of the I number). Q - HMT by parameter $\theta=\left[P_{S_{i}}(m), \xi_{i, \rho(i)}^{m, r}, \mu_{i, m}, \sigma_{i, m}^{2}\right]$ for the model, and to describe, which the probability of the root node aggregation function $\xi_{i, \rho(i)}^{m, r}=P_{S_{i} \mid s_{\rho(i)}}\left(m \mid s_{\rho(i)}=r\right), P_{S_{i}}(m)$ are given in the,state of $\mathrm{r}$, sub-state $s_{\rho(i)}$ in the conditional probability of $\mathrm{m}, S_{i}$ is the state transition probability.

Figure 1 shows the two-dimensional wavelet domain hidden markov tree model diagram.

\section{Simulation Results and Analysis}

In order to verify this section NWDM method of denoising and recovery effect, a series of simulation experiments are carried out using Matlab. The original image is 256 x 256 Barb gray image. Before and after the experiment adopted the hybrid difference scheme discretization, variable stepsize $h=1$, the space for the diffusion function

$$
\text { - } g(s)=\frac{1}{1+(s / k)^{2}}
$$

Wavelet selection of wavelet and biorthogonal wavelet bior4.4 original image in different intensity of zero mean white Gauss noise. Using P - M - lambda, CLMC model, NWDM model for peak signal-tonoise ratio (PSNR) biggest restored image. From figure 1 to figare 3 is to use three different methods for noise pollution after the noise reduction processing the the images of the restored image effect. Table 1 shows the different noise intensity the use of P - M - lambda model, CLMC model and NWDM model proposed by this section, filtering the noise image respectively, the restored image PSNR (dB)

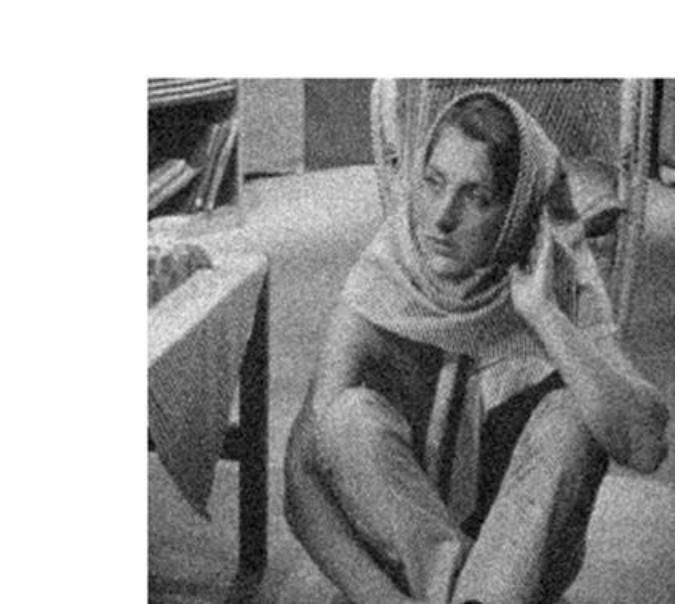

(a) The Gaussian image

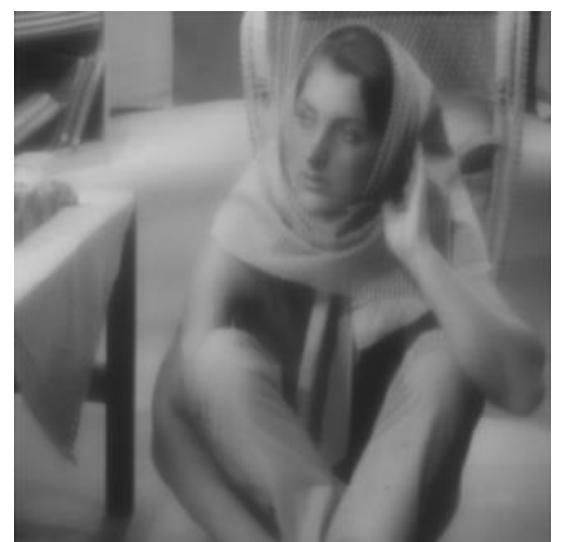

(b) P- M - lambda model With noise 


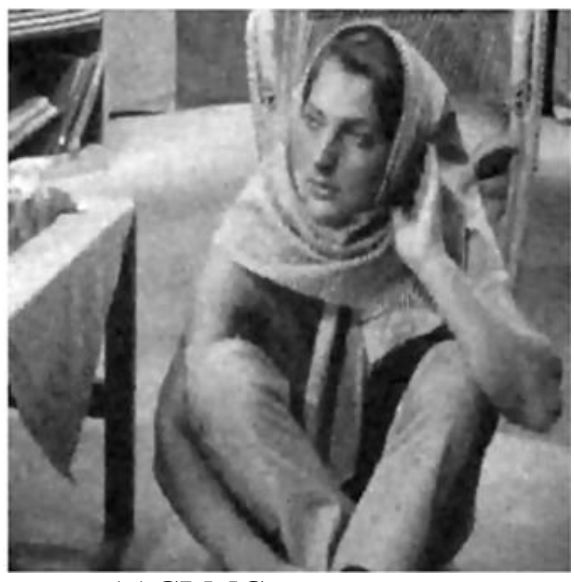

(c)CLMC

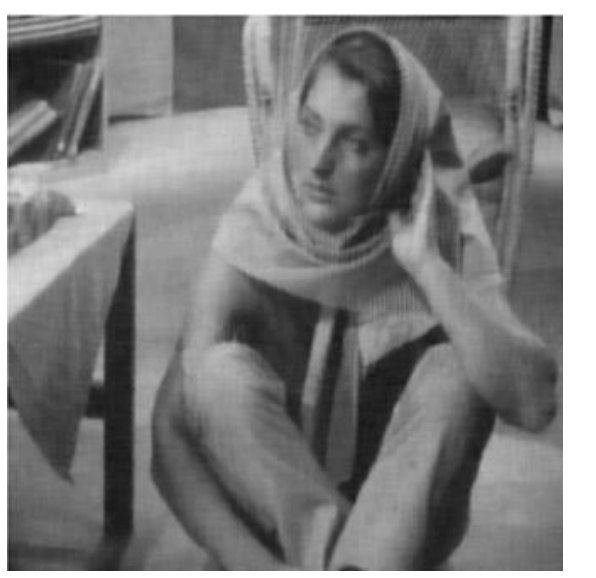

(d) NWDM

Figure 1. Noise Intensity $\sigma^{2}=15$,Image Restoration Effect using Three Diffusion Models

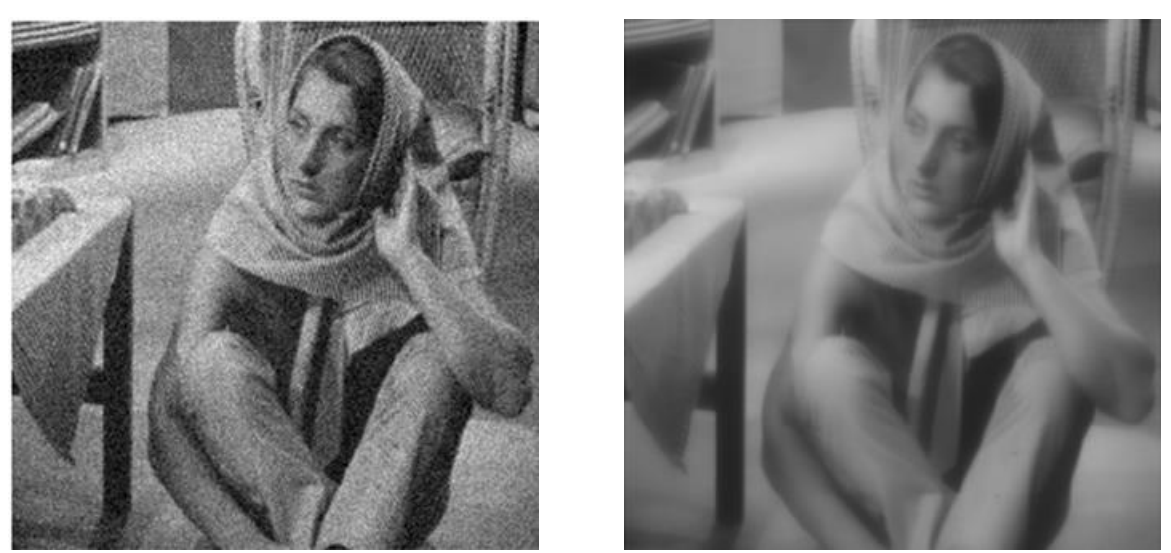

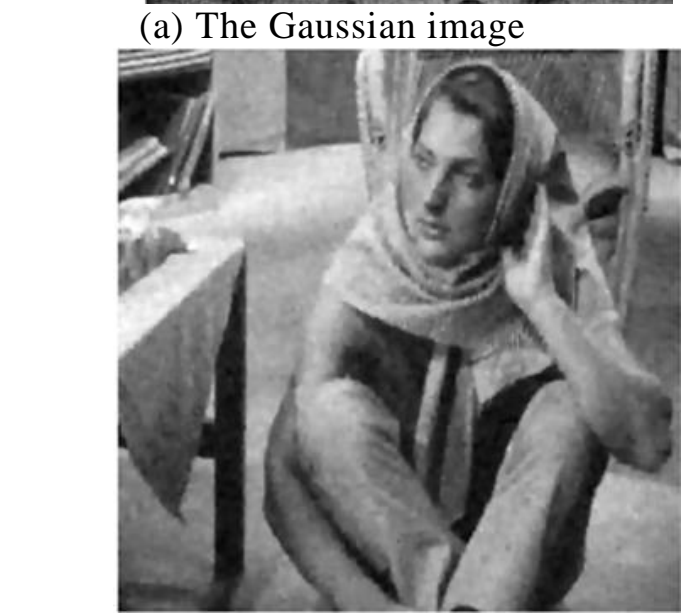

(c)CLMC (b) P- M - lambda model With noise

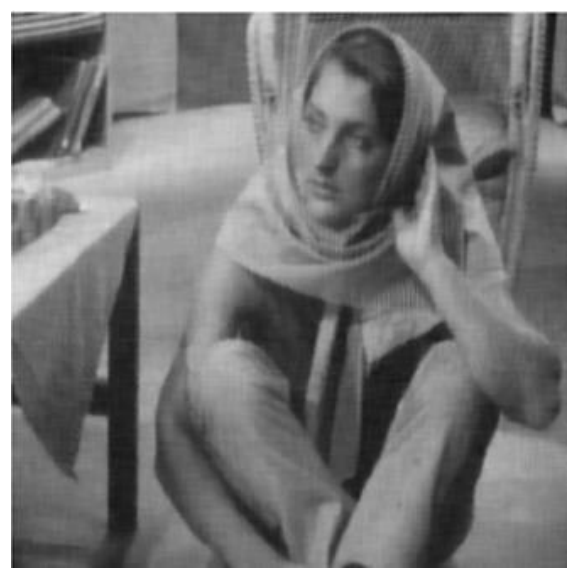

(d)NWDM

Figure 2. Noise Intensity $\sigma^{2}=\mathbf{2 0}$,Image Restoration Effect using Three Diffusion Models 


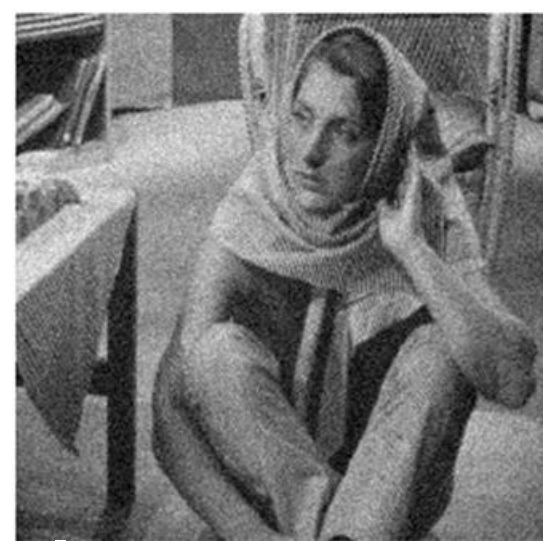

(a) The Gaussian image

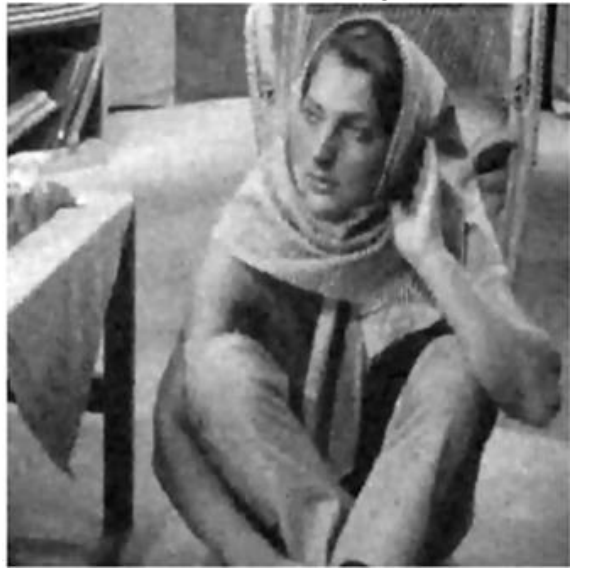

(c)CLMC

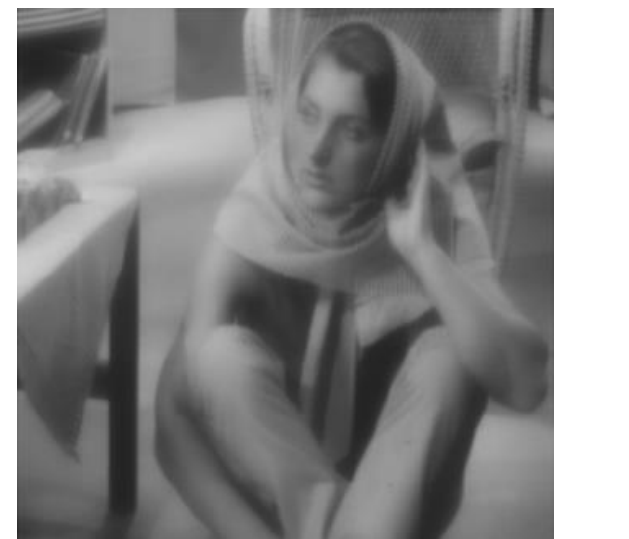

(b) P- M - lambda model With noise

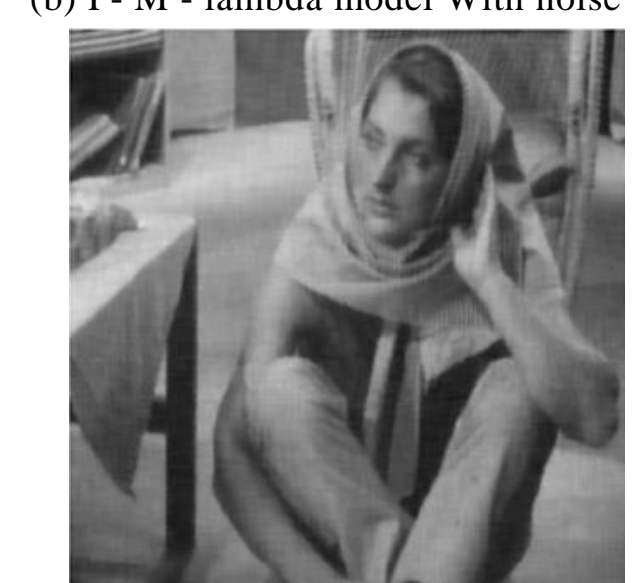

(d)NWDM

Figure 3. Noise Intensity $\sigma^{2}=25$,image Restoration Effect using Three Diffusion Models

The experiment results analysis:

Represented by P - M model of nonlinear diffusion equation of the diffusion of power from the image gradient, the cime of the end of diffusion and gradient threshold decides the effect of diffusion filtering, but for now, the selection of these two parameters are generally determine according to experience, not a can combine images geometry structure and the characteristics of the noise threshold parameter and the terminating time adaptive selection method. Look from image diffusion dynamic process, the introduction of a control mechanism in the diffusion equation, the control mechanisms can describe the image structure feature, to adjust the purpose of the diffusion behavior of the diffusion equation and constraint; Think in terms of the total energy of the diffusion process image changes, the introduction of numerical fidelity term, keep the evolution image and the original image similarity. This is the basic idea of this ZhangFangFa.

By the data under different noise intensity, using NWDM model to simulate the noise of image filtering after the image of the highest peak signal-to-noise ratio, second CLMC model, P - M minimum lambda model. CLMC model is higher than P - M filtering image signal to noise ratio, is the role of the gaussian smoothing. And NWDM model is using the multi-scale multi-resolution analysis of wavelet transform features, more useful than other methods can better describe the image information and noise, to better implement the signal-noise separation. NWDM model because of the introduction of the fidelity term, on the one hand, can make the image evolution process is accelerated, on the other hand, keeps evolving images and the degree of approximation of the original image. 
See from Figure 1 to Figure 3, after filtering NWDM model to get the best image visual effect. The experimental results show the effectiveness and superiority of NWDM model.

\section{Conclusion}

Anisotropic diffusion mechanism in introduced in this paper, on the basis of analyzed emphatically represented by $\mathrm{P}$ - M model of nonlinear diffusion model of diffusion filtering principle, and their respective characteristics and problems. Secondly, in-depth analysis of the nonlinear diffusion model, the threshold and termination mechanism of combining image geometric structure feature and visual information (gradient, brightness, contrast, structural information), was proposed based on nonlinear diffusion of threshold parameter selection and optimal diffusion time scale estimation method, this method is simple and can beself adapted to the image. Third, in view of the existing nonlinear diffusion filtering model the diffusion coefficient depends on the gradient and the problem that the susceptible to noise interference, presents a fidelity term used in image denoising and restoration contain nonlinear wavelet diffusion model (NWDM), theoretical analysis and experimental results show that NWDM compared to other diffusion model while denoifing can keep image edges and details characteristics, image visual effect is better.

\section{References}

[1] A. F. Abdelnour and I. W. Selesnick, "Nearysymmetrî or hogonal wavelet bases", Proc. IEEE Int. Conf. Acoust.,peech, Signal Processing (ICASSP), (2001) May.

[2] G. Beylkin, "On the representation of operators in basés of compactly supported wavelets", SlAM .J. Numer.Anal., vol. 29, no. 6, (1992) pp. 1716-1740.

[3] J. A. Bilmes, "A gentle tutorial of the EM/algopithm and its application to parameter estimation for gaussian mixture and hideren) markov models", International Computer Science Institute, TR-97-021, (1998).

[4] T. Bulow, "Hyperomplex spectral signal representations for the processing And analysis of images .Ph.D. dissertation", Christian AlbrechtsUniv Kiel, Germany, (1999).

[5] M. Bahri, "Construction quarterfion-valued wavelets", Mathematica, vol. 26, no. 1, (2010), pp. 107-114.

[6] B. Bayraktar and M. Analoui, Image denoising via fundamental anisotropic diffusion and wavelet shrinkage: a comparative st(dy) computational imaging II", Proceeding ofSPIE-IS and T Electric Imaging, (2004), vol. 5299, pp. 387-398.

[7] M. Bahri, R. Ashing and R. Vaillancourt, "Two-dimensionalquaternion wavelet transforms Applied Mathematics and Computation", vol. 218, (2011), pp. 10-21.

[8] T. D. Bui and G. Y. Chen, "Translation-invariant denoising using multiwavelets", IEEE Trans. Signal Processing, vol. 46, no. 12, (1998), pp. 3414-3420.

[9] A. G. Bruceand H. Y. Gao, "Understanding waveShrink: variance and bias estimation.Biometrika, vol. 83, no. 4, (1996), pp. 727-745.

[10] W. Bender, D. Gruhl and N. Morimoto, "Techniques for Data Hidding", Proceedings of the SPIE 2420, storage and retrieval for image and video database, vol. 3, (1995), pp. 164-173.

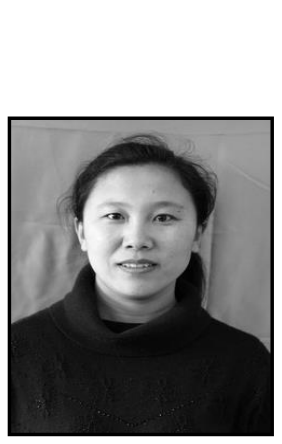

\section{Author}

Zhao Xiaofeng, she obtained her Bachelor's Degree of Science from Shandong Normal University In 2004 and obtained Master's Degree from Shandong University in 2011. She is currently researching on Digital Image Processing Theory and Application. 
International Journal of Multimedia and Ubiquitous Engineering Vol.9, No.7 (2014)

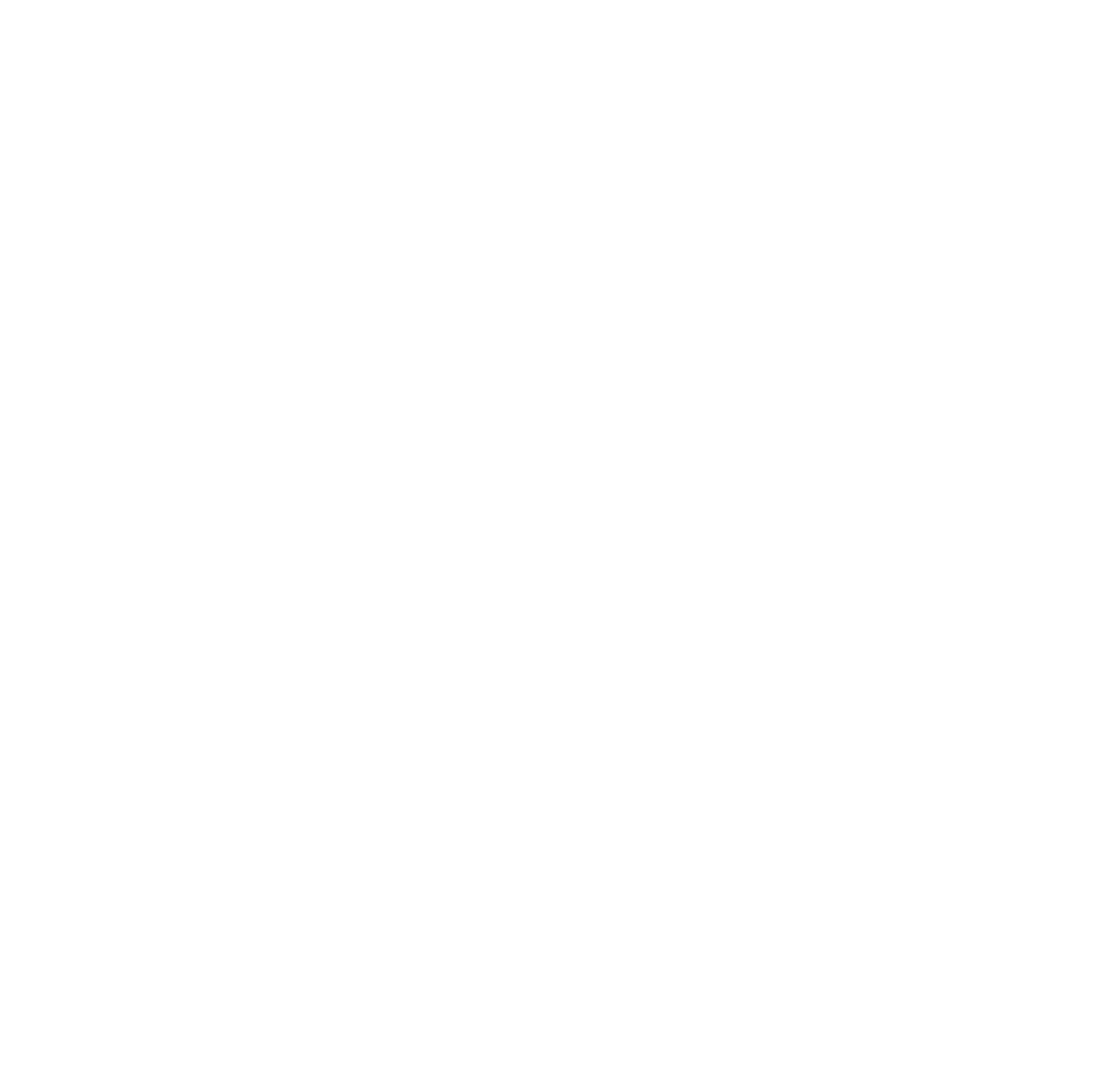

